\title{
miR-455 targets FABP4 to protect human endometrial stromal cells from cytotoxicity induced by hydrogen peroxide
}

\author{
WENBO TANG, OUJING CHEN, FENGXIANG YAO and LINING CUI \\ Department of Obstetrics and Gynecology, Ningbo First Hospital, Ningbo, Zhejiang 315000, P.R. China
}

Received July 13, 2018; Accepted April 4, 2019

DOI: $10.3892 / \mathrm{mmr} .2019 .10727$

\begin{abstract}
Oxidative stress and dysregulation of antioxidant systems are associated with various complications in pregnancy. Endometriosis is a common gynecologic disease that affects women of reproductive age. Recent studies have indicated that oxidative stress may be involved in the pathophysiology of endometriosis. It has been reported that microRNAs can regulate the cellular response to oxidative stress, and mounting evidence indicates that fatty acid binding protein 4 (FABP4) plays an essential role in the regulation of systemic redox capacity. In the present study, we demonstrated that miR-455 is a putative FABP4-targeting miRNA. A luciferase activity assay revealed that miR-455 can successfully bind to the 3'-UTR of FABP4. Overexpression of miR-455 led to the downregulation of FABP4 at both the mRNA and protein levels in a human endometrial stromal cell line. Then, the roles of miR-455 and FABP4 in oxidative stress induced by hydrogen peroxide $\left(\mathrm{H}_{2} \mathrm{O}_{2}\right)$ in human endometrial stromal cells were examined. We found that ectopic expression of miR-455 protected cells from damage caused by $\mathrm{H}_{2} \mathrm{O}_{2}$. Further investigation found that forced expression of miR-455 reduced the level of reactive oxygen species (ROS) and malondialdehyde (MDA), while the activities of superoxide dismutase (SOD), catalase (CAT) and glutathione peroxidase (GSH-Px) were promoted. Silencing of FABP4 also generated cytoprotective effects against $\mathrm{H}_{2} \mathrm{O}_{2}$ in human endometrial stromal cells. Moreover, overexpression FABP4 abrogated the miR-455-mediated antioxidative stress effects in cells. Taken together, we propose that miR-455 protects human endometrial stromal cells from oxidative stress at least partly via regulation of FABP4.
\end{abstract}

\section{Introduction}

Endometriosis is a disorder characterized by implantation and growth of endometrial tissue outside the uterine cavity.

Correspondence to: Dr Lining Cui, Department of Obstetrics and Gynecology, Ningbo First Hospital, 59 Liuting Road, Ningbo, Zhejiang 315000, P.R. China

E-mail: cuiliningnb@sohu.com

Key words: miR-455, FABP4, endometrial stromal cells, oxidative stress, $\mathrm{H}_{2} \mathrm{O}_{2}$
It has been estimated that approximately $10-15 \%$ of women of reproductive age are affected by endometriosis (1). To date, the etiology of endometriosis has yet to be elucidated; many theories have been proposed, but none of them have been substantiated (2-4). Recently, substantial evidence indicates that oxidative stress, which is defined as an imbalance between reactive oxygen species (ROS) and antioxidants, may be involved in the pathophysiology of endometriosis by triggering a general inflammatory response in the peritoneal cavity (5). Consequently, ROS are likely to be associated with different pregnancy-related disorders, such as spontaneous abortions, fetal growth restriction and preeclampsia. Therefore, targeting oxidative imbalance may be applicable as an effective therapeutic strategy against endometriosis.

MicroRNAs (miRNAs) are a group of small non-coding RNAs that regulate the expression of genes at the posttranscriptional level via inhibition of protein translation and decay of mRNAs (6). It is well documented that miRNAs are involved in various biological processes including the response to oxidative stress. For instance, a recent study showed that miR-182-5p inhibits oxidative stress via targeting Toll-like receptor 4 (TLR4) in macrophages (7). Forced expression of miR-210 was found to lead to the reduction of BNIP3 and protected rat myocardial cells from oxidative stress induced by $\mathrm{H}_{2} \mathrm{O}_{2}$ (8). miR-29b was found to regulate oxidative stress via targeting SIRT1 in ovarian cancer cells (9). miR-455 has been found to be involved in a variety of cancers, and it was shown that miR-455 could protect osteoblasts from oxidative stress via activation of the $\mathrm{Nrf} 2$ signaling pathway $(10,11)$. However, whether miR-455 can alleviate oxidative stress in endometrial stromal cells remains unknown.

Fatty acid binding protein 4 (FABP4), also known as adipocyte FABP (aP2), is a small 15-kDa lipid chaperone that participates in various biological processes such as glucose and lipid homeostasis, inflammation and intracellular fatty acid trafficking (12). A recent study has shown that FABP4-1macrophages exhibit diminished ROS production (13). In contrast, it was also found that FABP4 plays a cytoprotective role against oxidative and ER stress in adipocytes and that the knockdown of FABP4 causes an increase in cellular ROS levels (14). These findings indicate that FABP4 plays an essential role in the regulation of oxidative stress, and thus, further investigation is warranted in terms of its role in endometriosis.

In the present study, we identified miR-455 as a putative FABP4-targeting miRNA. Forced expression of miR-455 
protected endometrial stromal cells from cytotoxicity induced by $\mathrm{H}_{2} \mathrm{O}_{2}$. Moreover, silencing of FABP4 generated protective effects similar to those of miR-455, which were abrogated by the ectopic expression of FABP4 in endometrial stromal cells.

\section{Materials and methods}

Cell culture and transfection. Immortalized human endometrial stromal cells (HESCs) were purchased from Applied Biological Materials Ltd.. Cells were cultured in a 1:1 mixture of Dulbecco's modified Eagle's medium (DMEM) (Gibco ${ }^{\mathrm{TM}}$; Thermo Fisher Scientific, Inc.) and Ham's F-12 medium $\left(\right.$ Gibco $^{\mathrm{TM}}$; Thermo Fisher Scientific, Inc.) supplemented with $10 \%$ fetal calf serum (FCS) (HyClone; GE Healthcare), $100 \mathrm{U} / \mathrm{ml}$ penicillin and streptomycin (HyClone; GE Healthcare). Cells were cultured in a humidified atmosphere with $5 \% \mathrm{CO}_{2}$ at $37^{\circ} \mathrm{C}$. miR-455 mimics (miR-455), negative control mimics (miR-NC), siRNA against FABP4 (si-FABP4), negative control siRNA (si-NC) and pcDNA3.1-FABP4 (pcFABP4) were purchased from GenePharm. Transfection was performed using Lipofectamine 2000 (Thermo Fisher Scientific, Inc.) according to the manufacturer's instructions. Unless indicated, the dose of $\mathrm{H}_{2} \mathrm{O}_{2}$ used in this study was $20 \mu \mathrm{M}$.

Quantitative real-time PCR ( $q P C R)$. Total RNA was extracted from cells using TRIzol reagent (Thermo Fisher Scientific, Inc.) according to the manufacturer's instructions. Briefly, $1 \mu \mathrm{g}$ of total RNA was used to synthesize cDNA using a TIANScript II cDNA First Chain Synthesis kit (Tiangen Biotech Co., Ltd). Then, qPCR was performed with a miScript SYBR-Green PCR kit (Qiagen, Inc.) on an ABI 7500 system (PE Applied Biosystems $^{\mathrm{TM}}$; Thermo Fisher Scientific, Inc.). GAPDH and U6 were used as internal controls. The thermocycling conditions were as follows: $95^{\circ} \mathrm{C}$ for $1 \mathrm{~min}$, then 40 cycles of $95^{\circ} \mathrm{C}$ for $15 \mathrm{sec}, 55^{\circ} \mathrm{C}$ for $30 \mathrm{sec}$ and $70^{\circ} \mathrm{C}$ for $30 \mathrm{sec}$. The expression levels in tissues and cells were calculated using the $2^{-\Delta \Delta \mathrm{Cq}}$ method (15).

Dual-luciferase reporter assay. Two oligonucleotide pairs including FABP4 3'UTR sequences of both the mutant and wild-type sequences were synthesized by Genepharm. After annealing, the oligonucleotides were inserted into the pmirGLO vector (Fig. S1) (Promega, Madison, WI, USA). For luciferase assays, the cells were co-transfected with the corresponding vectors and miR-455a mimics using Lipofectamine 2000 (Thermo Fisher Scientific, Inc.) according to the manufacturer's protocol. Then, $24 \mathrm{~h}$ after transfection, the firefly and Renilla luciferase activities were detected consecutively using a Dual-Luciferase Kit (Promega Corp.). The relative luciferase activity was normalized to that of Renilla luciferase.

Cell viability assay. A CCK-8 assay was performed to measure cell viability after treatment. Briefly, cells (5,000 cells/well) were seeded in a 96-well plate. After treatment, $10 \mu \mathrm{l}$ of Cell Counting Kit-8 solution (CCK-8; cat. no. c0038, Beyotime Institute of Biotechnology) was added, and the optical density (OD) value of each well was measured at a wavelength of $595 \mathrm{~nm}$ using an ELISA microplate reader. Wells without cells served as blanks. The experiments were performed in triplicate and were repeated at least three times.
Apoptosis assay. For apoptosis detection by flow cytometry, the cells were stained with propidium iodide (PI) and Annexin V-FITC (cat. no. v13242; Invitrogen; Thermo Fisher Scientific, Inc.); the fluorescence was then determined by a BD FACSVia ${ }^{\mathrm{TM}}$ flow cytometry system (BD Biosciences).

Caspase-3/7 activity assay. The activity of caspase-3/7 was measured using a Caspase-Glo 3/7 Assay kit (cat. no. g8090, Promega) according to the manufacturer's protocol. Briefly, $100 \mu \mathrm{l}$ of caspase-3/7 reagent was added to each well followed by incubation for $1 \mathrm{~h}$ at room temperature. Luminescence was measured as the absorbance at $405 \mathrm{~nm}$. Caspase-3/7 activity was indicated as a percentage of the untreated control. Three independent experiments were performed.

Western blot analysis. After treatment, cells were collected and lysed in RIPA buffer (Beyotime Institute of Biotechnology). Equal amounts of protein extracts $(20 \mu \mathrm{g})$ were subjected to $12 \%$ sodium dodecyl sulfate-polyacrylamide gel electrophoresis (SDS-PAGE) and transferred to a polyvinylidene difluoride (PVDF) membrane (Millipore). The membrane was blocked with $5 \%$ skim milk for $1 \mathrm{~h}$ at room temperature. Then, the membrane was incubated with the primary antibody overnight at $4^{\circ} \mathrm{C}$. The following primary antibodies were used: FABP4 (cat. no. ab66682; Abcam), actin (cat. no. ab179467; Abcam), and caspase-3 (cat. no. 9662; Cell Signaling Technology). The primary antibodies were diluted at the ratio of 1:1,000 in TBST. Following three washes in TBST for $15 \mathrm{~min}$ each, the membranes were incubated with a goat anti-rabbit horseradish peroxidase-conjugated secondary antibody (cat. no. 7074; Cell Signaling Technology) for $1 \mathrm{~h}$ at room temperature. The secondary antibody was diluted at the ratio of 1:10,000 in TBST. The results were visualized using the Super Signal Chemiluminescent Substrate (Pierce; Thermo Fisher Scientific, Inc.) according to the manufacturer's instructions. Protein bands were quantified by densitometric analysis using Quantity One software v4.6.6 (Bio-Rad Laboratories).

Determination of ROS, $L D H, C A T, G S H-P x, M D A$, and SOD activity. For the assessment of reactive oxygen species (ROS), DCF-DA (Thermo Scientific) was used as an ROS probe, as previously described (16). After different treatments, the cells were incubated with $5 \mu \mathrm{M}$ DCF-DA for $30 \mathrm{~min}$ at $37^{\circ} \mathrm{C}$. The stained cells were then analyzed by flow cytometry (FACS Caliber, BD Biosciences). Lactate dehydrogenase (LDH) activity was measured using an LDH ELISA kit (cat. no. MAK066, Sigma-Aldrich; Merck KGaA) according to the manufacturer's instructions. The activities of catalase (CAT), glutathione peroxidase (GSH-Px), malondialdehyde (MDA) and superoxide dismutase (SOD) were determined using commercially available colorimetric assay kits (cat. nos. ab83464, ab239727, ab118970, ab211096, respectively; Abcam) according to the manufacturer's protocols.

Statistical analysis. Data are expressed as the mean \pm standard deviation (SD) and were analyzed using SPSS 18.0 (SPSS, Inc.). Statistical comparisons between different groups were measured using Student's t-test or a one-way analysis of variance (ANOVA) with post-hoc Tukey's test. $\mathrm{P}<0.05$ was considered to indicate a statistically significant result. 
A
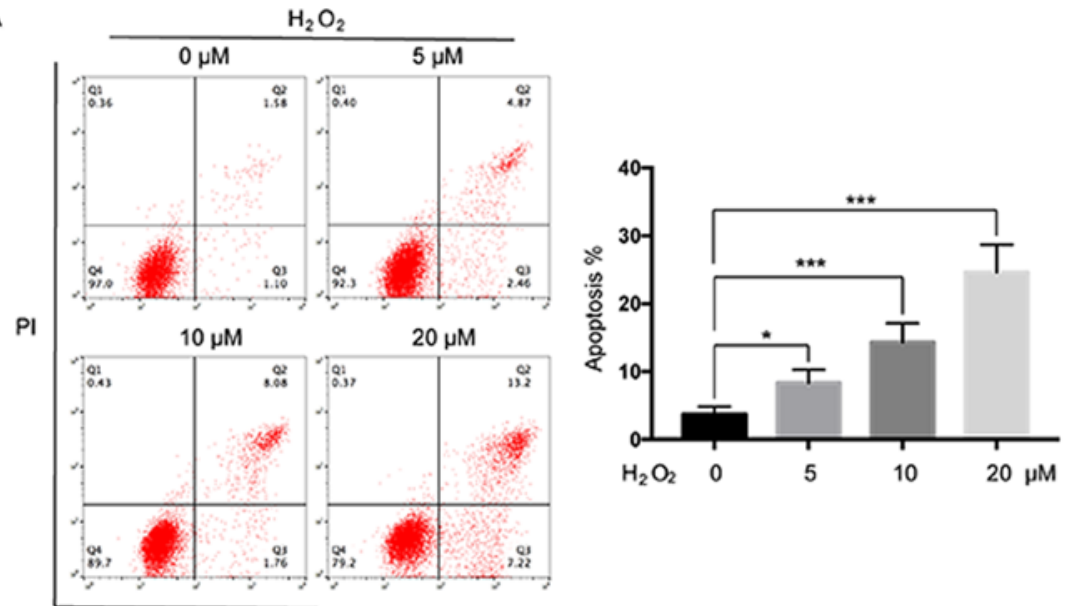

Annexin V-FITC

B

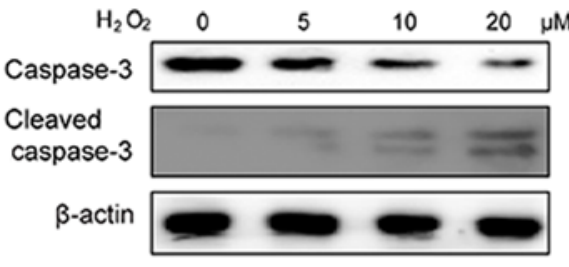

C

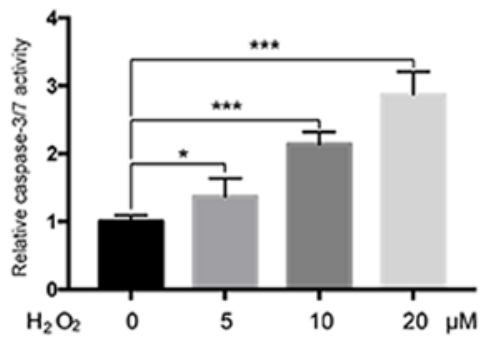

D
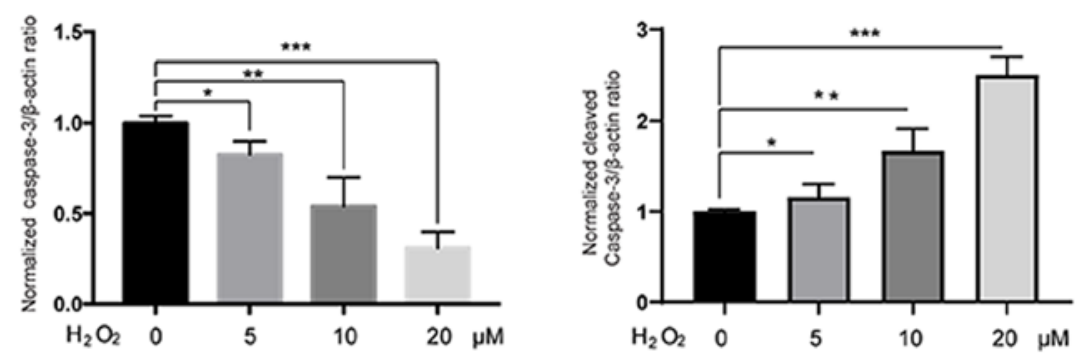

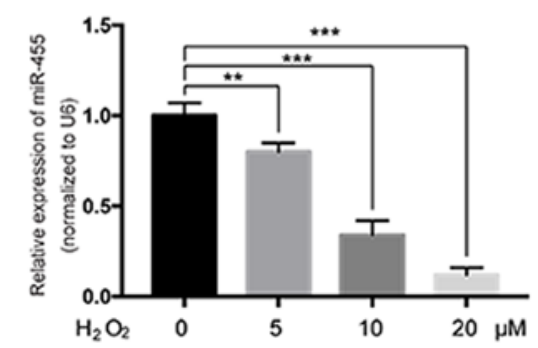

Figure $1 . \mathrm{H}_{2} \mathrm{O}_{2}$ induces apoptosis and affects the expression of miR-455 in HESCs. (A) Flow cytometry analysis of the apoptosis rates in HESCs exposed to various doses of $\mathrm{H}_{2} \mathrm{O}_{2}$ for $24 \mathrm{~h}$. (B) HESCs were treated with various concentrations of $\mathrm{H}_{2} \mathrm{O}_{2}$ for $24 \mathrm{~h}$, and then, cellular lysates were collected and subjected to western blot analysis with the caspase-3 antibody. The histograms (right) show the densitometric analysis of the caspase-3 and cleaved caspase-3 western blot results. (C) HESCs were treated with various concentrations of $\mathrm{H}_{2} \mathrm{O}_{2}$, after which total cellular lysates were collected and subjected to a caspase-3/7 activity assay. (D) HESCs were treated with various concentrations of $\mathrm{H}_{2} \mathrm{O}_{2}$ for $24 \mathrm{~h}$, and then, the levels of miR-455 were measured by qPCR. All data are shown as the mean \pm SD of three independent experiments. ${ }^{*} \mathrm{P}<0.05,{ }^{* *} \mathrm{P}<0.01$ and ${ }^{* * *} \mathrm{P}<0.001$. HESCs, human endometrial stromal cells; $\mathrm{H}_{2} \mathrm{O}_{2}$, hydrogen peroxide.

\section{Results}

Hydrogen peroxide induces apoptosis and decreases the expression of miR-455 in human endometrial stromal cells. First, HESCs were treated with various doses of $\mathrm{H}_{2} \mathrm{O}_{2}$ for $24 \mathrm{~h}$ after which the apoptosis rates were measured. As shown in Fig. 1A, flow cytometric analysis revealed that treatment with $\mathrm{H}_{2} \mathrm{O}_{2}$ significantly induced apoptosis of HESCs in a dose-dependent manner. Western blot analysis also demonstrated that $\mathrm{H}_{2} \mathrm{O}_{2}$ treatment led to a decrease in pro-caspase-3 and an increase in cleaved caspase- 3 in a dose-dependent manner in HESCs (Fig. 1B). Moreover, a caspase-3/7 activity assay further confirmed that treatment with $\mathrm{H}_{2} \mathrm{O}_{2}$ significantly increased the activation of caspase- $3 / 7$ in a dose-dependent manner in HESCs (Fig. 1C). Then, miR-455 levels were examined in $\mathrm{H}_{2} \mathrm{O}_{2}$-treated HESCs, and the results showed that miR-455 levels were significantly reduced in HESCs in a dose-dependent manner following $\mathrm{H}_{2} \mathrm{O}_{2}$ treatment. Taken together, these data suggest that miR-455 is likely negatively correlated with apoptosis induced by $\mathrm{H}_{2} \mathrm{O}_{2}$ in HESCs.

Overexpression of miR-455 protects HESCs from $\mathrm{H}_{2} \mathrm{O}_{2}$-induced apoptosis. Next, we explored whether miR-455 plays a role in $\mathrm{H}_{2} \mathrm{O}_{2}$-induced apoptosis of HESCs. A CCK-8 assay showed that the $\mathrm{H}_{2} \mathrm{O}_{2}$-induced reduction in cell viability was significantly reversed by overexpression of miR-455 mimics in HESCs (Fig. 2A). Flow cytometric analysis also indicated that upregulation of miR-455 significantly reduced the apoptosis induced by $\mathrm{H}_{2} \mathrm{O}_{2}$ in HESCs (Fig. 2B and Fig. S2). Western blot analysis found that cleavage of caspase- 3 induced by $\mathrm{H}_{2} \mathrm{O}_{2}$ was diminished by overexpression of miR-455 in HESCs (Fig. 2C). Furthermore, the caspase-3/7 activity 
A

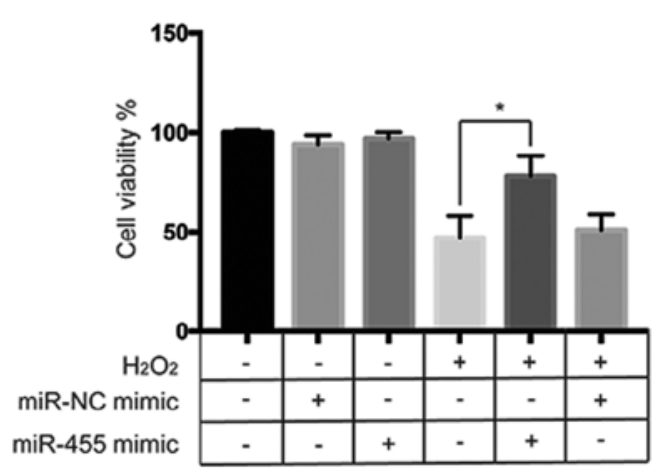

C
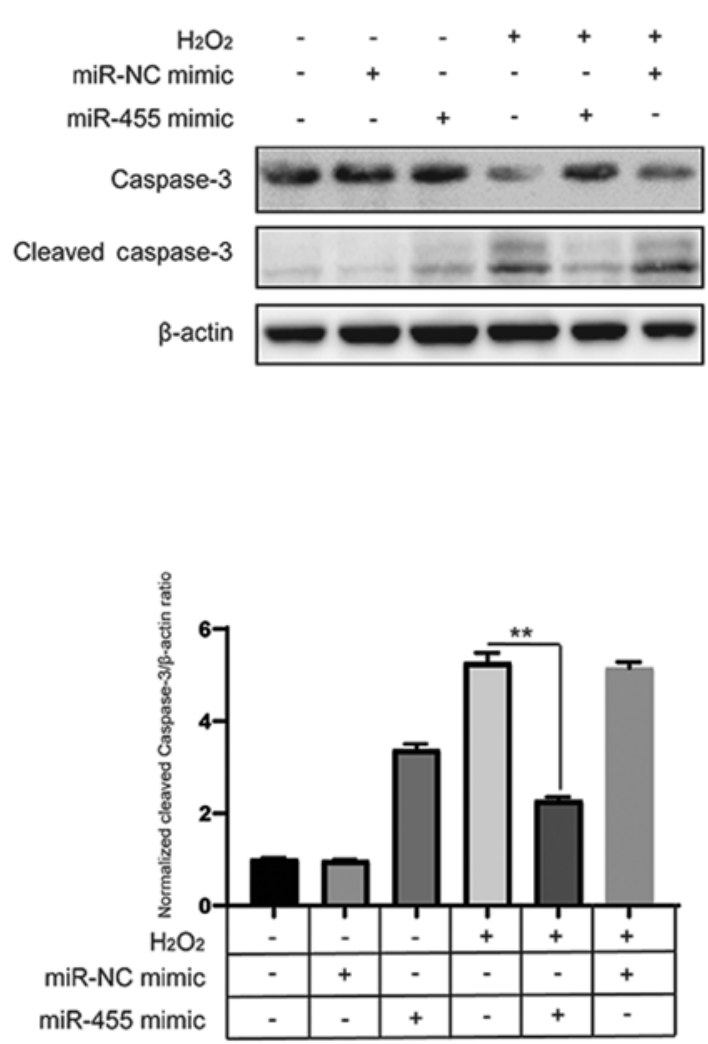

B
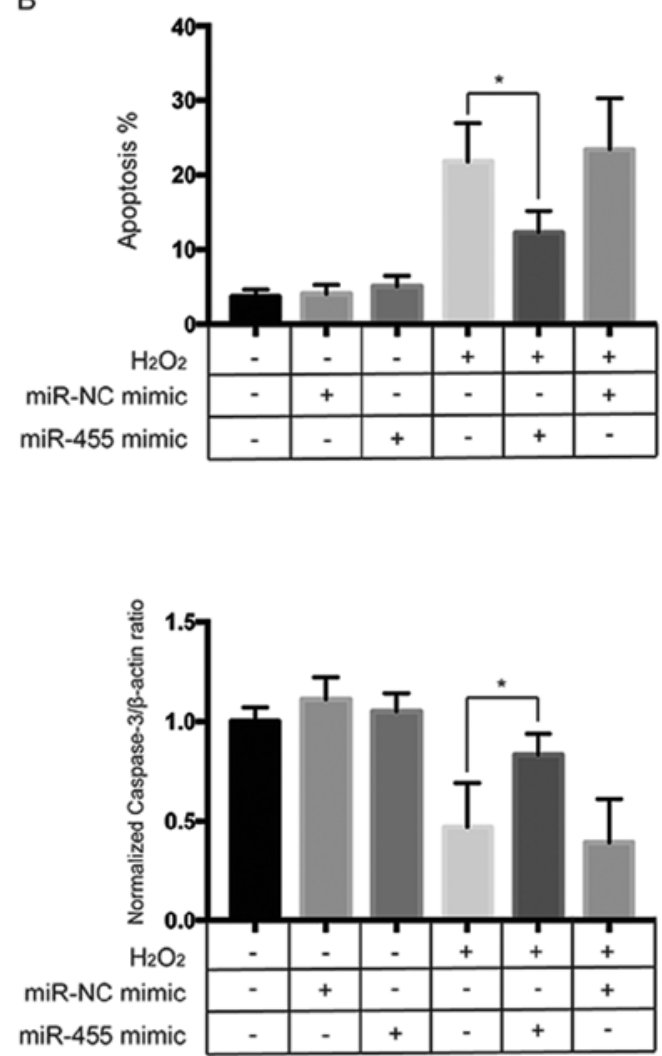

D

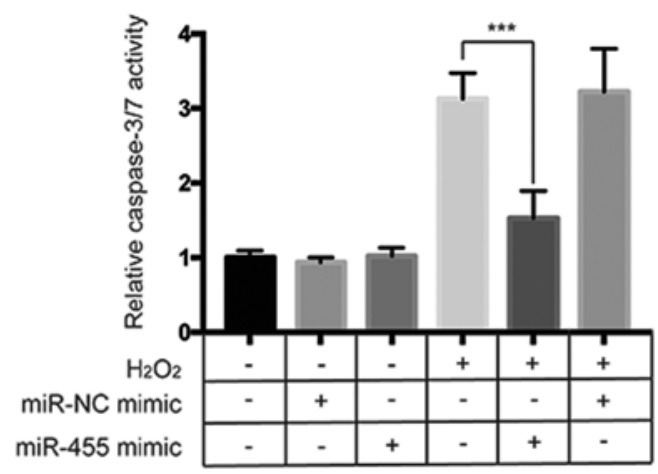

Figure 2. miR-455 inhibits $\mathrm{H}_{2} \mathrm{O}_{2}$-induced apoptosis of HESCs. (A) HESCs were treated as indicated for $24 \mathrm{~h}$, and then a CCK-8 assay was performed to detect cell viability. (B) The apoptosis rate of HESCs following the different treatments. (C) HESCs were treated as indicated; subsequently, total cellular lysates were subjected to western blot analysis with the caspase-3 antibody. The histograms shows the densitometric analysis of the caspase-3 (right panel) and cleaved caspase-3 (bottom panel) western blot results. (D) HESCs were treated as indicated. Then, the relative caspase-3/7 activities were determined. All data are shown as the mean $\pm \mathrm{SD}$ of three independent experiments. ${ }^{*} \mathrm{P}<0.05 ;{ }^{* *} \mathrm{P}<0.01{ }^{* * * *} \mathrm{P}<0.001$. HESCs, human endometrial stromal cells; $\mathrm{H}_{2} \mathrm{O}_{2}$, hydrogen peroxide.

assay also revealed that activation of caspase- $3 / 7$ induced by $\mathrm{H}_{2} \mathrm{O}_{2}$ was repressed by upregulation of miR-455 (Fig. 2D). These results indicate that miR-455 plays a protective role in $\mathrm{H}_{2} \mathrm{O}_{2}$-induced apoptosis of HESCs.

miR-455 alleviates the oxidative stress induced by $\mathrm{H}_{2} \mathrm{O}_{2}$ in HESCs. We then investigated the effects of miR-455 on oxidative stress induced by $\mathrm{H}_{2} \mathrm{O}_{2}$ in HESCs. First, we examined the effect of miR-455 on the intracellular ROS levels induced by $\mathrm{H}_{2} \mathrm{O}_{2}$. As shown in Fig. 3A, exposure to $\mathrm{H}_{2} \mathrm{O}_{2}$ significantly increased the ROS levels, which were significantly reduced by overexpression of miR-455. MDA is a degradation product of membrane lipid oxidation that can serve as an indicator of oxidative damage (17). To further investigate the antioxidant function of miR-455, the levels of MDA were measured after treatment. As indicated in Fig. $3 \mathrm{~B}, \mathrm{H}_{2} \mathrm{O}_{2}$ treatment led to a significant upregulation of intracellular MDA levels, while overexpression of miR-455 significantly diminished this effect. Furthermore, the activities of endogenous antioxidative enzymes such as SOD, CAT and GSH-Px were also determined. As shown in Fig. 3C-E, $\mathrm{H}_{2} \mathrm{O}_{2}$ significantly decreased the SOD, CAT and GSH-Px activities, which were partly reversed by upregulation of miR-455. Taken together, these findings confirmed that miR-455 alleviates oxidative stress in HESCs. 
A

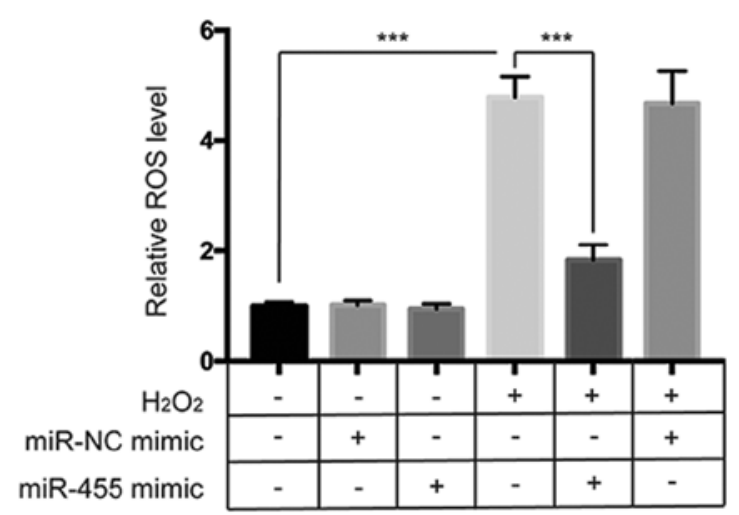

C

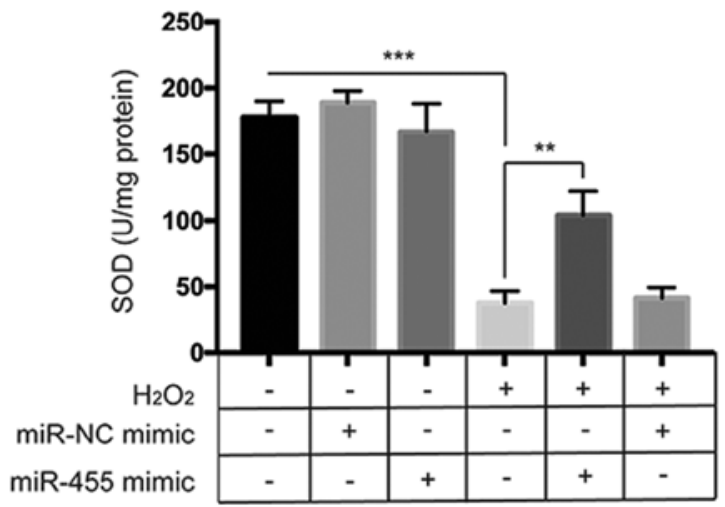

B

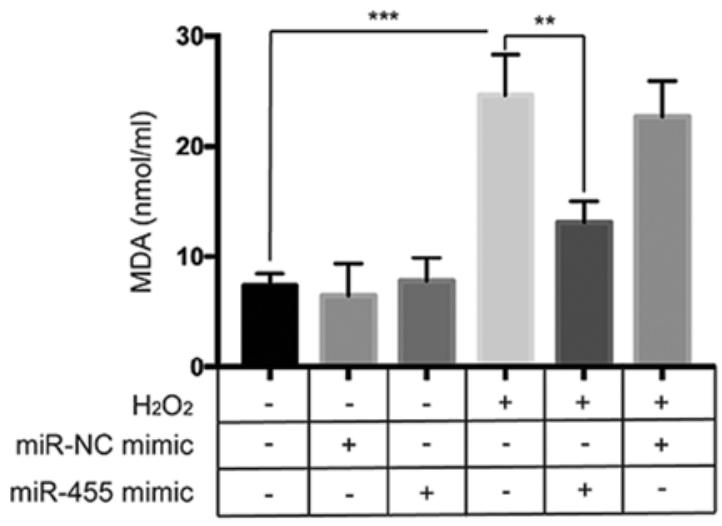

D

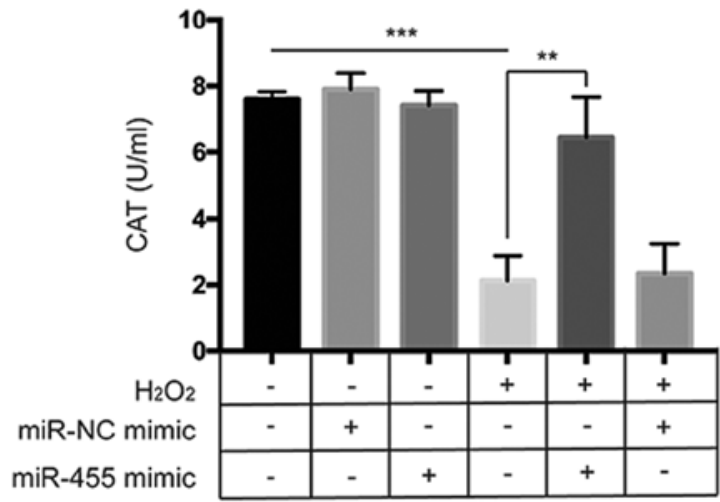

E

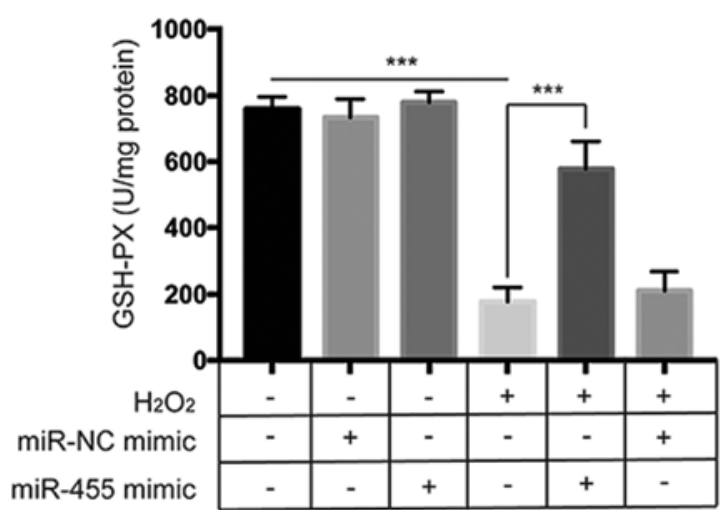

Figure 3. miR-455 alleviates oxidative stress in HESCs. (A) HESCs were treated as indicated, and then intracellular ROS levels were measured by flow cytometry. (B) HESCs were treated as indicated, after which the MDA activity was assessed. (C) HESCs were treated as indicated, after which the SOD activity was assessed. (D) HESCs were treated as indicated, after which CAT activity was assessed. (E) HESCs were treated as indicated, after which assessment of GSH-Px activity was determined. All data are shown as the mean \pm SD of three independent experiments. ${ }^{* *} \mathrm{P}<0.01$ and ${ }^{* * *} \mathrm{P}<0.001$. HESCs, human endometrial stromal cells; ROS, reactive oxygen species; MDA, malondialdehyde; SOD, superoxide dismutase; CAT, catalase; GSH-Px, glutathione peroxidase.

FABP4 is negatively regulated by miR-455. To identify potential targets of miR-455, the bioinformatic tools TargetScan (www.targetscan.org) and StarBase V2.0 (starbase. sysu.edu.cn) were used. It was shown that miR-455 putatively targets the 3'-UTR of FABP4 mRNA (Fig. 4A). Furthermore, we performed a luciferase reporter assay to further confirm whether miR-455 can directly target the 3'-UTR region of FABP4. Both the FABP4 wild-type (WT) 3'UTR containing the miR-455 binding site and a mutated FABP4 3'-UTR sequence were cloned into luciferase reporter vectors, which were co-transfected with miR-NC or a miR-455 mimic. qPCR analysis revealed that transfection of cells with miR-455 mimics successfully increased the endogenous level of miR-455 compared with the negative control (Fig. 4B). As indicated in Fig. 2C, overexpression of miR-455 significantly $(\mathrm{P}<0.01)$ decreased the activity of luciferase encoded by a gene containing FABP4 3'-UTR WT, while it did not affect the activity of luciferase encoded by a gene containing FABP4 

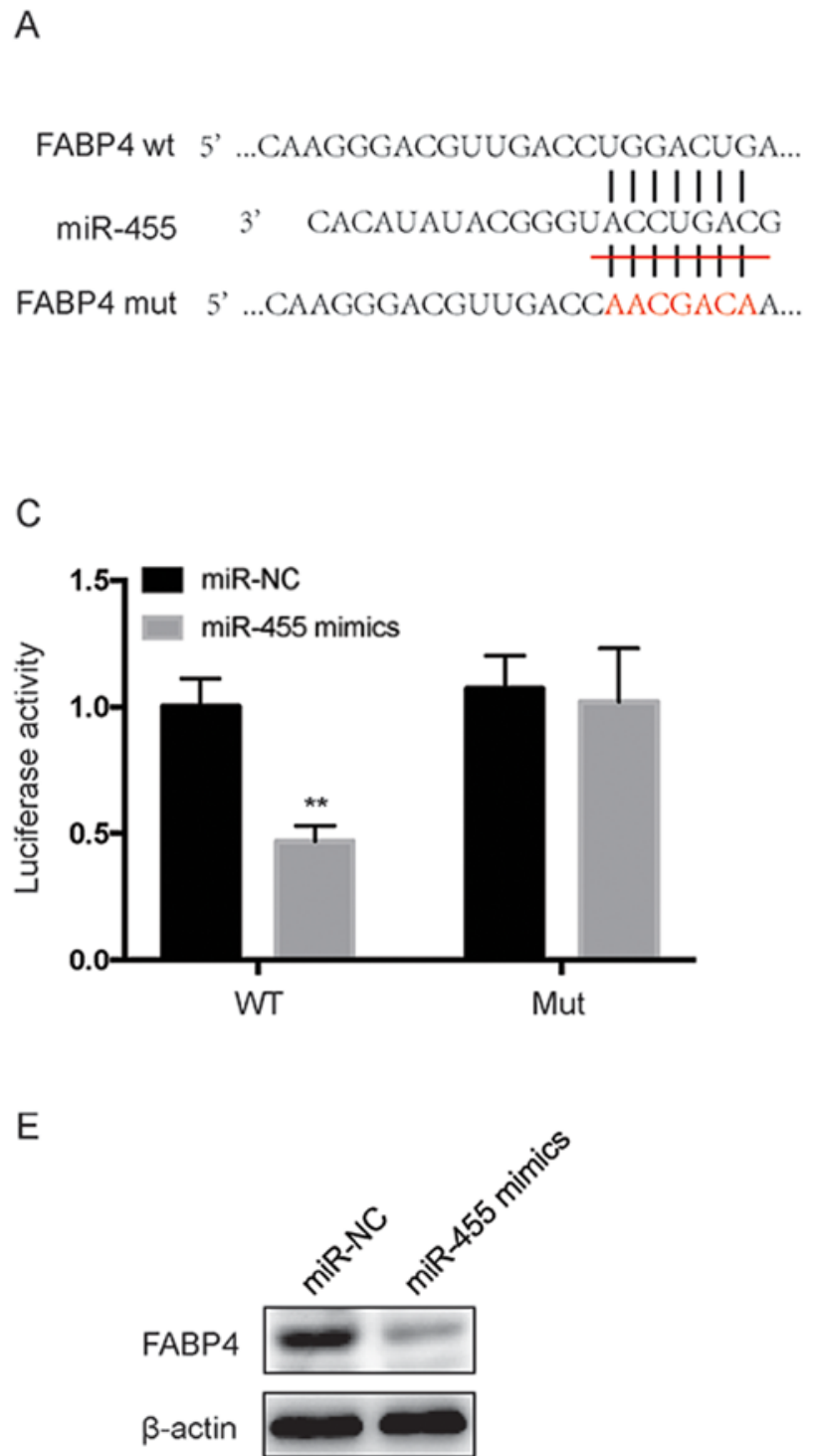

B

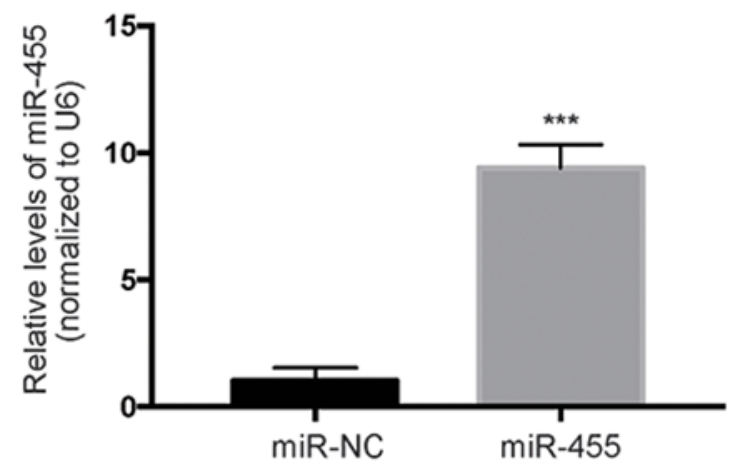

D
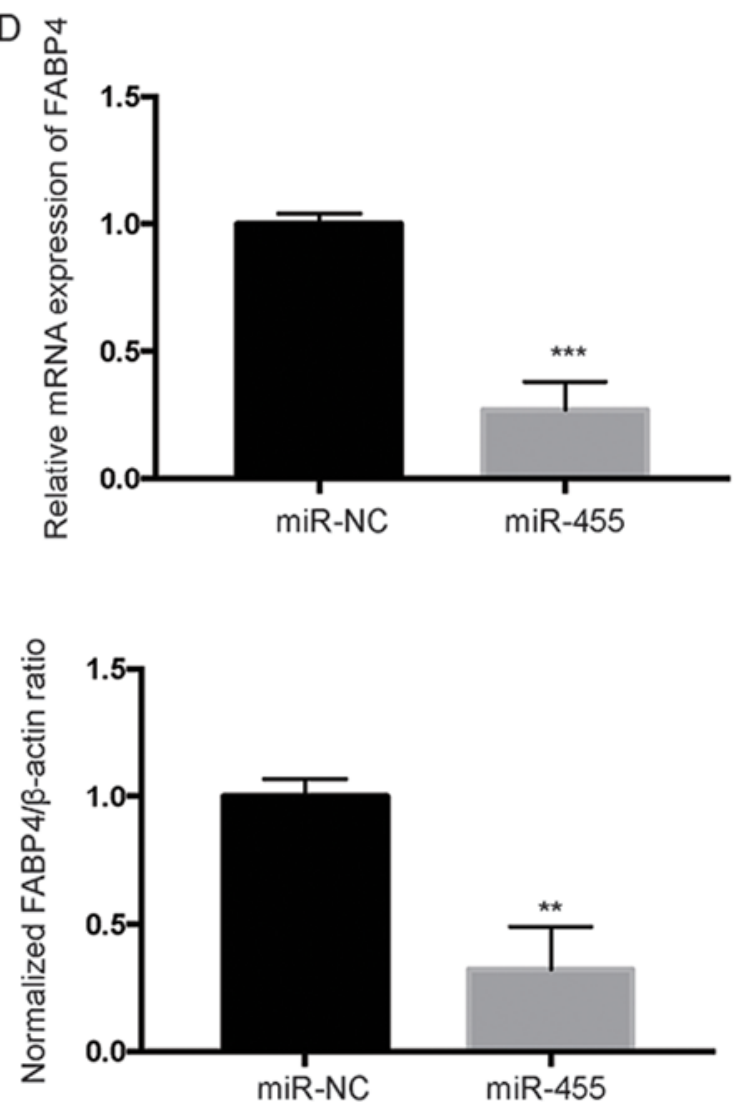

Figure 4. FABP4 is a direct target of miR-455 in HESCs. (A) The predicted miR-455 binding site within the FABP 3'-UTR and a mutated version generated by site-directed mutagenesis. The mutant nucleotides of the FABP4 3'-UTR are labeled in red. (B) HESCs were transfected with miR-455 mimics or miR-NC. Twenty-four hours after transfection, miR-455 expression was measured by qPCR. (C) A luciferase assay was performed using HESCs that were co-transfected with miR-NC or miR-455 and a luciferase reporter containing the full length of FABP4 3'-UTR wild-type (WT) or a mutant type (mut). Luciferase activities were measured $24 \mathrm{~h}$ after transfection. (D) qPCR showed that the mRNA levels of FABP4 were decreased in HESCs after transfection with miR-455. (E) Western blot analysis of FABP4 in HESCs after transfection with miR-455. All data are shown as the mean \pm SD of three independent experiments. ${ }^{* *} \mathrm{P}<0.01$ and ${ }^{* * *} \mathrm{P}<0.001$. FABP4, fatty acid binding protein 4 ; HESCs, human endometrial stromal cells.

3'UTR Mut. Further investigation revealed that miR-455 overexpression significantly decreased the mRNA and protein levels of FABP4 in HESCs (Fig. 4D and E). Collectively, these data indicate that FABP4 is a direct target of miR-455 in HESCs.

Silencing of FABP4 is a phenocopy of the effect of miR-455. Next, to investigate the role of FABP4 in response to oxidative stress in HESCs, we used siRNA to specifically knock down the expression of FABP4. Twenty-four hours after transfection, the expression of FABP4 was measured by qPCR and western blot analysis. The results showed that transfection of cells with si-FABP4 successfully repressed the expression of FABP4 (Fig. 5A and B). CCK-8 assays showed that siFABP4 protects
HESCs from the $\mathrm{H}_{2} \mathrm{O}_{2}$-induced decrease in cell viability (Fig. 5C). Flow cytometric analysis of apoptosis showed that $\mathrm{H}_{2} \mathrm{O}_{2}$-induced apoptosis was reversed by siFABP4 (Fig. 5D and Fig. S3). Furthermore, western blot analysis and caspase-3/7 activity assay revealed that the $\mathrm{H}_{2} \mathrm{O}_{2}$-induced activation of caspase- 3 was diminished by siFABP4 (Fig. 5E and F).

Upregulation of FABP4 diminishes the protective effects of miR-455 against $\mathrm{H}_{2} \mathrm{O}_{2}$. To further investigate the role of FABP4, HESCs were transfected with miR-455 mimics and the FABP4 overexpression vector alone or in combination. qPCR and western blot analysis revealed that the FABP4 vector significantly upregulated the level of FABP4 (Fig. 6A and B). A CCK-8 
A

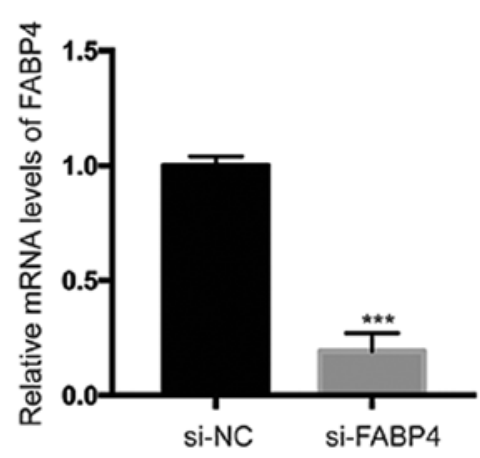

C

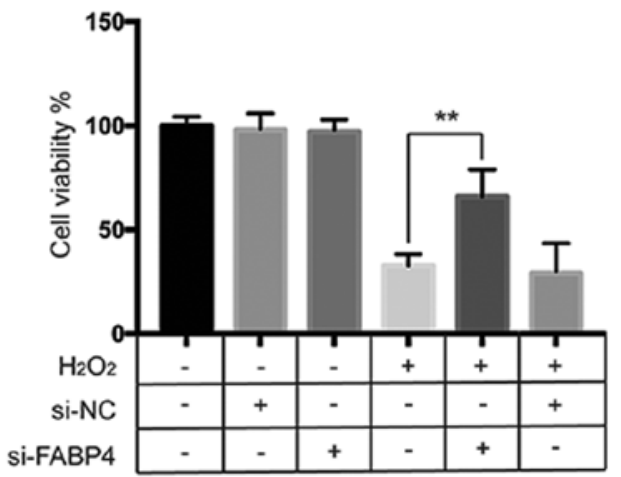

E
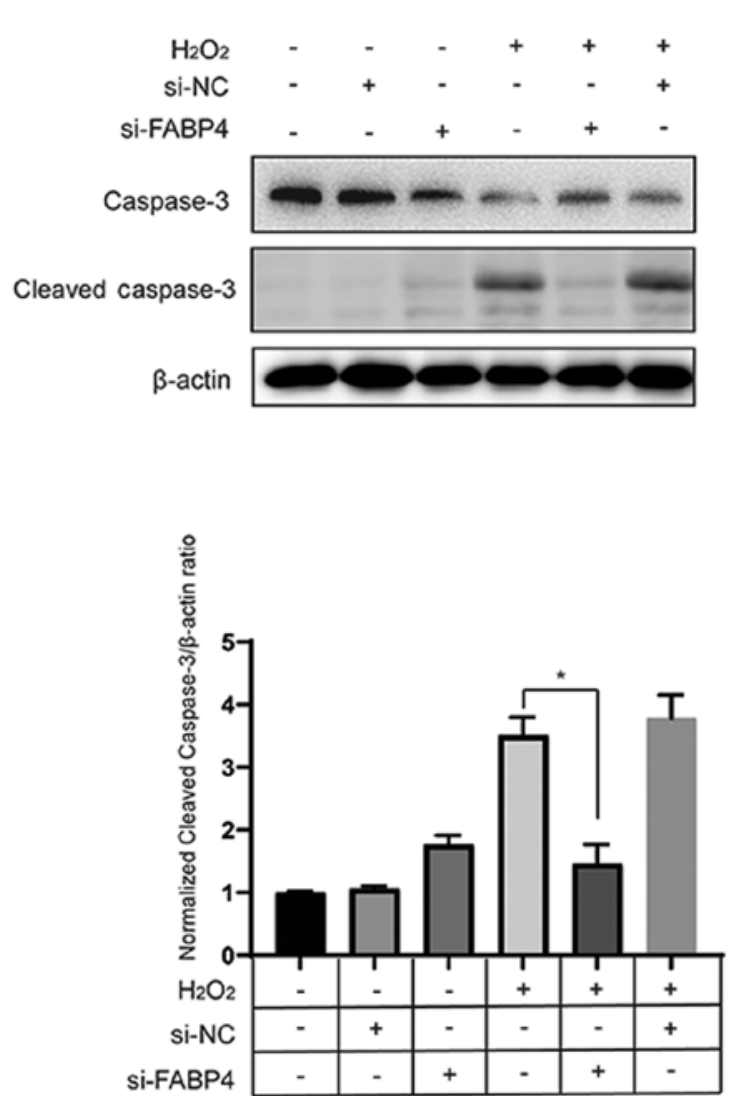

B

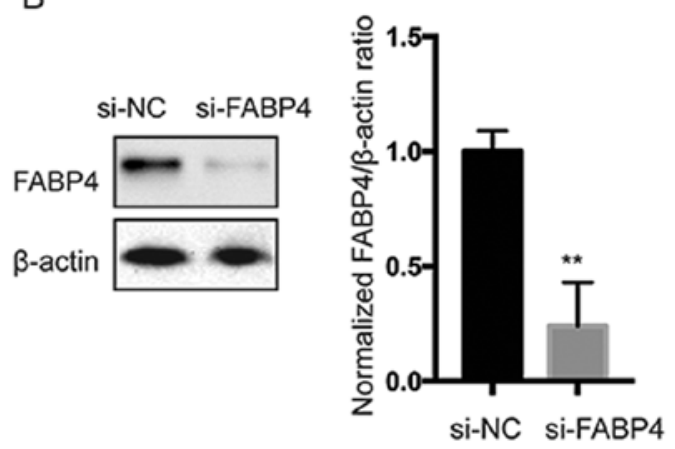

D
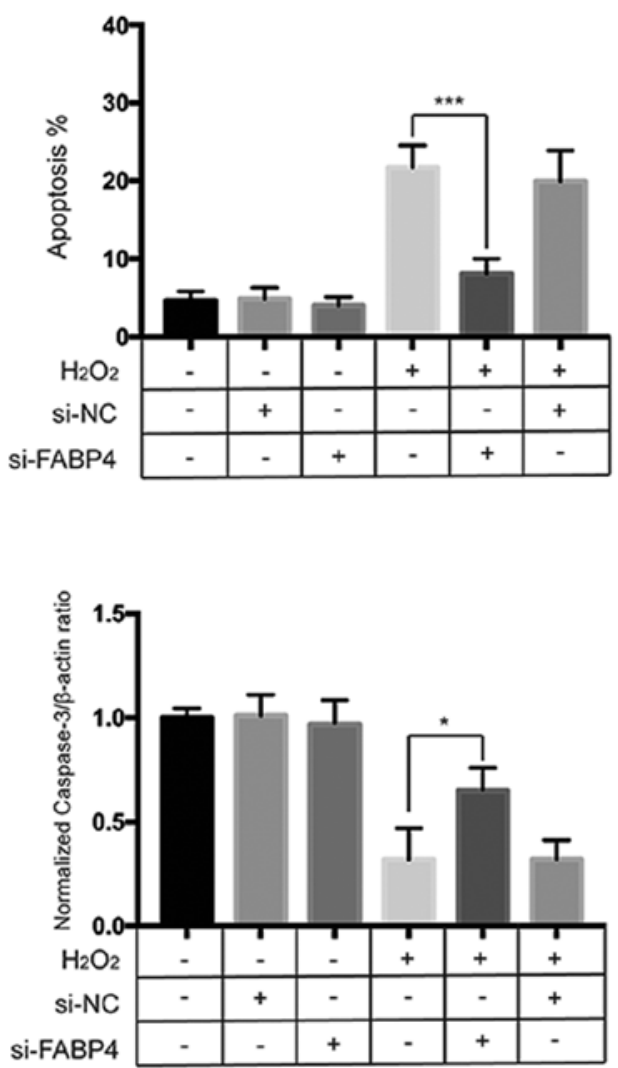

F

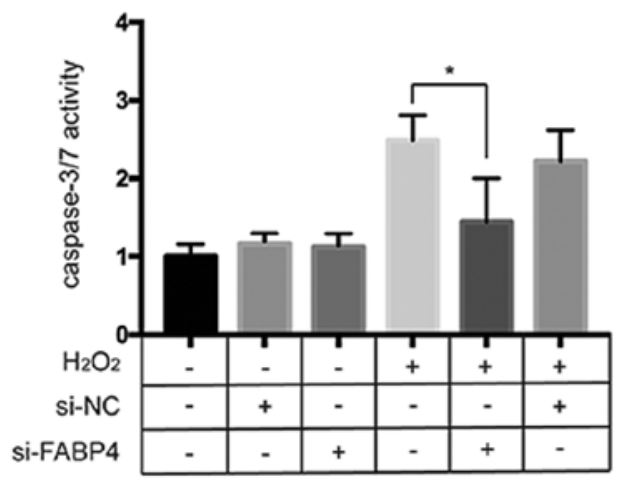

Figure 5. Silencing of FABP4 protects HESCs from apoptosis induced by $\mathrm{H}_{2} \mathrm{O}_{2}$ in HESCs. (A) HESCs were transfected with si-NC or si-FABP4 for 24 h, and then, the mRNA levels of FABP4 were measured by qPCR. (B) HESCs were transfected with si-NC or si-FABP4 for $24 \mathrm{~h}$. The protein levels of FABP4 were then assessed by western blot analysis. (C) HESCs were treated as indicated for $24 \mathrm{~h}$, which was followed by a CCK- 8 assay to detect cell viability. (D) The apoptosis rate of HESCs following the different treatments. (E) HESCs were treated as indicated, after which the total cellular lysates were subjected to western blot analysis with the caspase-3 antibody. The histogram shows the densitometric analysis of the caspase-3 (right panel) and cleaved caspase-3 (bottem panel) western blot results. (F) HESCs were treated as indicated, after which the relative caspase-3/7 activities were assayed. All data are shown as the mean \pm SD of three independent experiments. ${ }^{*} \mathrm{P}<0.05,{ }^{* *} \mathrm{P}<0.01$ and ${ }^{* * * *} \mathrm{P}<0.001$. FABP4, fatty acid binding protein $4 ; \mathrm{H}_{2} \mathrm{O}_{2}$, hydrogen peroxide; HESCs, human endometrial stromal cells. 
A

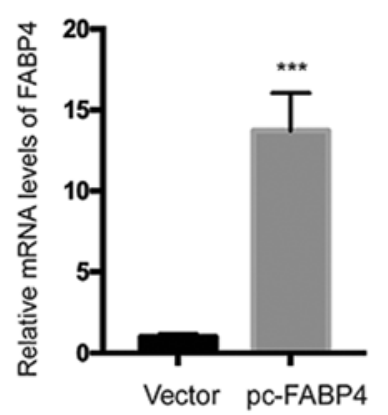

C

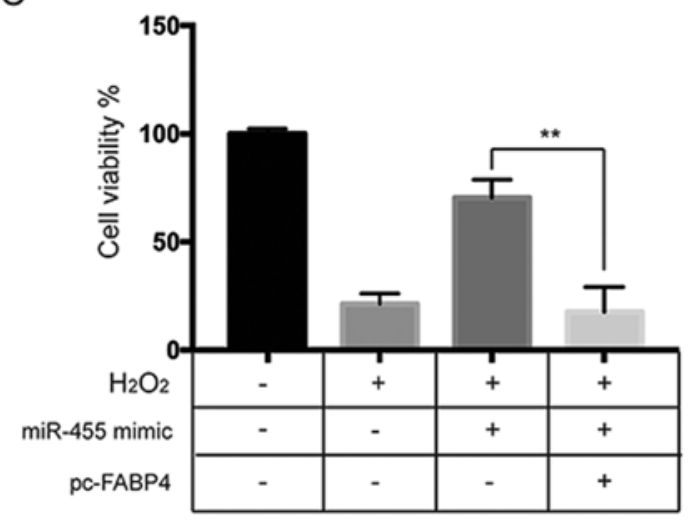

E

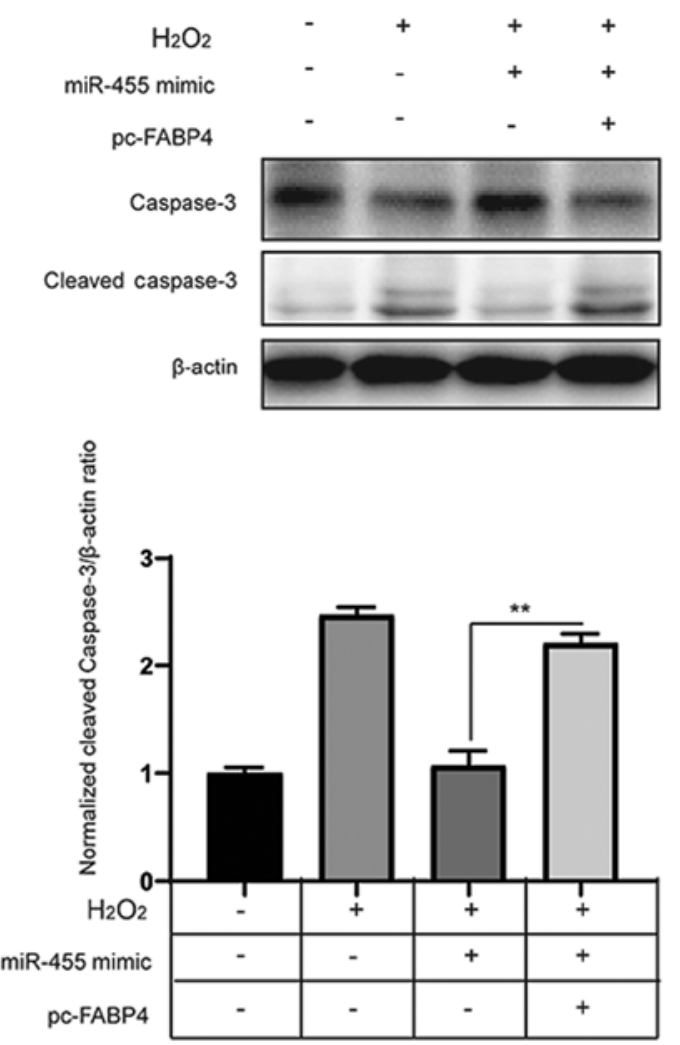

B
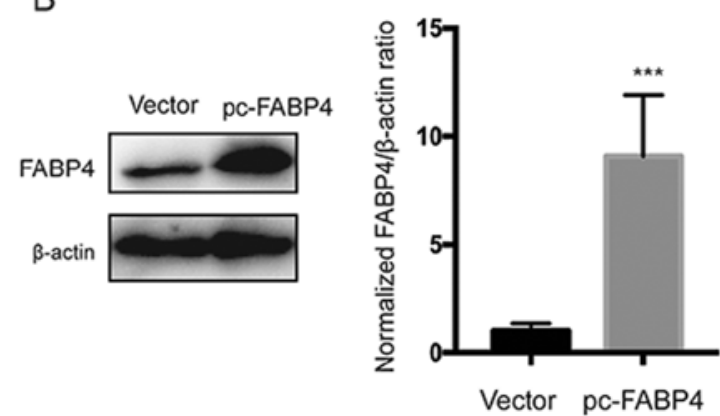

D
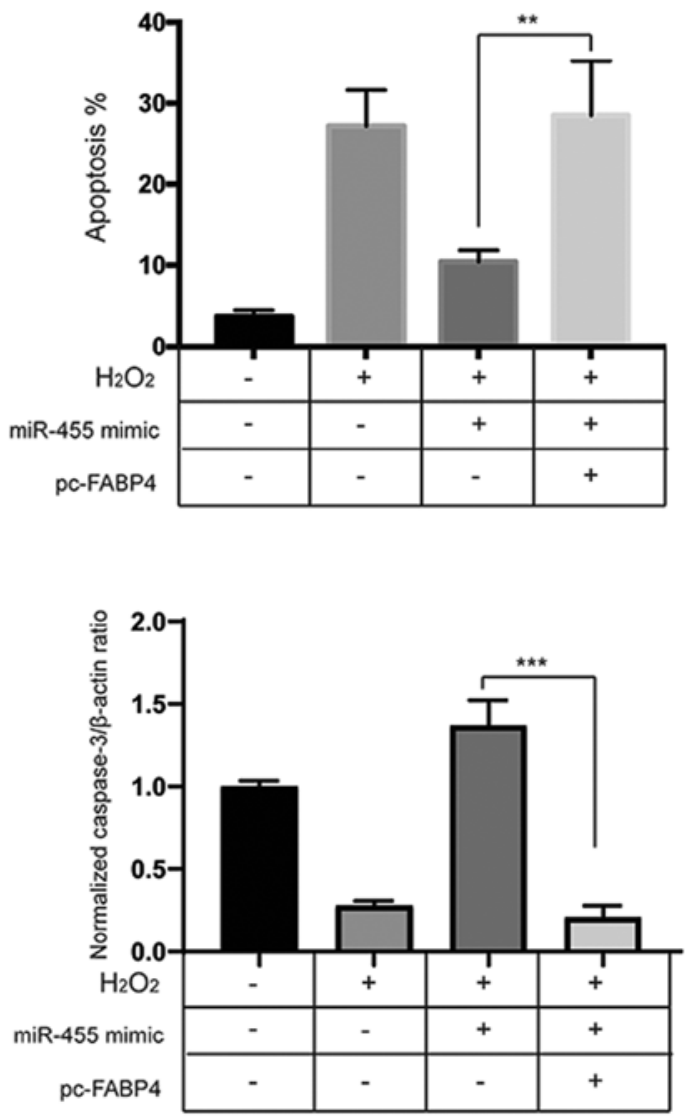

F

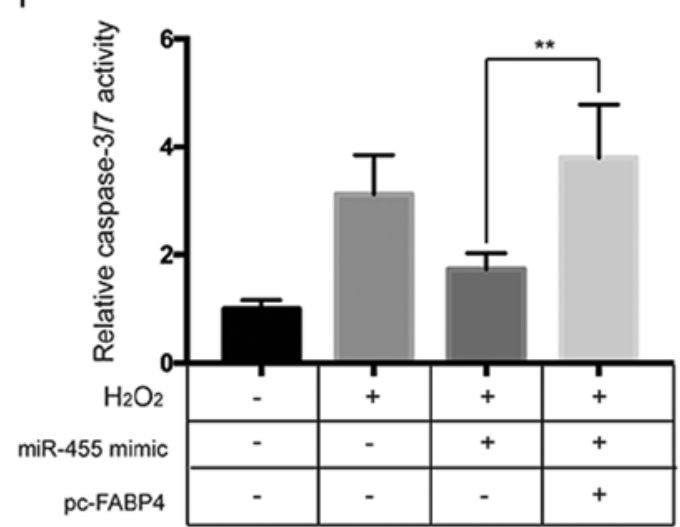

Figure 6. Ectopic expression of FABP4 abolishes the protective effects of miR-455 against $\mathrm{H}_{2} \mathrm{O}_{2}$. (A) qPCT analysis of FABP4 mRNA levels after ectopic expression of FABP4 for $24 \mathrm{~h}$. (B) Western blot analysis of FABP4 protein levels after ectopic expression of FABP4 for $24 \mathrm{~h}$. (C) HESCs were treated as indicated for $24 \mathrm{~h}$, and then, a CCK-8 assay was performed to detect cell viability. (D) The apoptosis rate of HESCs following the different treatments. (E) HESCs were treated as indicated, and then, total cellular lysates were subjected to western blot analysis with the caspase-3 antibody. The histogram shows the densitometric analysis of the caspase- 3 and cleaved caspase- 3 western blotresults. (F) HESCs were treated as indicated, after which the relative caspase-3/7 activities were assayed. All data are shown as the mean $\pm \mathrm{SD}$ of three independent experiments. ${ }^{* * *} \mathrm{P}<0.01$ and ${ }^{* * * *} \mathrm{P}<0.001$. FABP4, fatty acid binding protein 4 ; $\mathrm{H}_{2} \mathrm{O}_{2}$, hydrogen peroxide; HESCs, human endometrial stromal cells. 
assay showed that overexpression of FABP4 significantly diminished the protective effects of miR-455 against $\mathrm{H}_{2} \mathrm{O}_{2}$ (Fig. 6C) In addition, flow cytometric analysis showed that upregulation of FABP4 abrogated the protective role of miR-455 against apoptosis induced by $\mathrm{H}_{2} \mathrm{O}_{2}$ (Fig. 6D and Fig S4). Moreover, western blot analysis and the caspase-3/7 activity assay showed that overexpression of FABP4 significantly abrogated the inhibitory effect of miR-455 on the activation of caspase- 3 induced by $\mathrm{H}_{2} \mathrm{O}_{2}$ (Fig. 6E and F).

\section{Discussion}

In the present study, we examined the effects of miR-455 on oxidative stress in human endometrial stromal cells (HESCs). It was found that miR-455 was decreased by $\mathrm{H}_{2} \mathrm{O}_{2}$ in a dose-dependent manner. In addition, ectopic expression of miR-455 alleviated cellular damage induced by $\mathrm{H}_{2} \mathrm{O}_{2}$ in HESCs. It was also demonstrated that miR-455 inhibited $\mathrm{H}_{2} \mathrm{O}_{2}$-induced intracellular reactive oxygen species (ROS) production and the malondialdehyde (MDA) level; this effect was accompanied by significantly increased activities of catalase (CAT), superoxide dismutase (SOD) and glutathione peroxidase (GSH-Px). Moreover, fatty acid binding protein 4 (FABP4) was identified as a target of miR-455. Since oxidative stress is implicated in the pathophysiology of endometriosis as it causes a general inflammatory response in the peritoneal cavity, our findings suggest that miR-455 may be applied as part of a more optimized therapy for endometriosis.

$\mathrm{H}_{2} \mathrm{O}_{2}$ has been extensively applied as an inducer of oxidative stress in various in vitro models. $\mathrm{H}_{2} \mathrm{O}_{2}$ readily enters the cytoplasm and thereafter produces a more toxic hydroxyl radical that interacts with macromolecules such as lipids, proteins and DNA, which leads to cellular damage (18). To evaluate $\mathrm{H}_{2} \mathrm{O}_{2}$-related cellular damage, a CCK-8 assay and flow cytometry, which are widely used to evaluate the effect of pharmacological agents on cell proliferation and apoptosis, were performed. The results of our study revealed that $\mathrm{H}_{2} \mathrm{O}_{2}$ decreased the viability and increased the apoptosis of HESCs in a dose-dependent manner. Another significant finding was that $\mathrm{H}_{2} \mathrm{O}_{2}$ also decreased the expression of miR-455 in a dose-dependent manner. Although no previous study to date has reported the protective effect of miR-455 against oxidative stress in HESCs, $\mathrm{Xu}$ et al demonstrated that miR-455 targets cullin 3 to activate Nrf2 signaling and protects human osteoblasts from cellular damage induced by $\mathrm{H}_{2} \mathrm{O}_{2}$ (11). Moreover, in a recent study, Zhang et al showed that miR-455 protects osteoblasts from oxidative stress through activation of Nrf2/ARE signaling (10). Our results further confirmed that miR-455 overexpression largely relieved oxidative stress in HESCs and revealed that $\mathrm{H}_{2} \mathrm{O}_{2}$ inhibited HESC growth, at least in part, via downregulation of miR-455.

Many studies have shown that $\mathrm{H}_{2} \mathrm{O}_{2}$ exerts oxidative stress via inducing apoptosis in vitro $(19,20)$. Oxidative stress activates caspases, which are a group of cysteine proteases that act as death effector molecules, and after activation, they cleave various substrates in the cytoplasm or nucleus $(21,22)$. Two pathways lead to apoptosis, namely, the extrinsic and intrinsic pathways (22). The extrinsic pathway is initiated by caspase- 8 , while the intrinsic pathway is initiated by caspase-9 (22). The activation of caspase- 8 and/or caspase- 9 leads to the activation of caspase-3, which is the executioner caspase that can cleave different substrates and finally induce apoptosis $(21,22)$. Our study, which is in agreement with a previous study, indicated that a relatively low concentration of $\mathrm{H}_{2} \mathrm{O}_{2}$ induced apoptosis, which was accompanied by activation of caspase-3 in HESCs (23). In the present study, ectopic expression of miR-455 obviously restrained the $\mathrm{H}_{2} \mathrm{O}_{2}$-induced apoptosis in HESCs. Mounting evidence has confirmed that $\mathrm{H}_{2} \mathrm{O}_{2}$-induced accumulation of ROS, which can lead to cell damage and apoptosis, is implicated in the pathophysiology of endometriosis (24). The results of our study indicated that $20 \mu \mathrm{M}$ of $\mathrm{H}_{2} \mathrm{O}_{2}$ markedly increased the level of ROS, which was repressed by overexpression of miR-455. Moreover, overexpression of miR-455 markedly alleviated oxidative stress, as evidenced by the decreased MDA level and increased SOD, CAT and GSH-Px activities. Therefore, we concluded that miR-455 alleviated oxidative stress and thereby provides protection against $\mathrm{H}_{2} \mathrm{O}_{2}$ in HESCs.

FABP4, which has been identified as a direct downstream target of miR-455, belongs to the FABP family. It has been reported that FABP1, another member of the FABP family, functions as an antioxidant protein since it neutralizes free radicals through its methionine and cysteine amino acids (25). However, the role of FABP4 in the regulation of oxidative stress is still controversial. Many studies have shown that inhibition of FABP4 could suppress inflammation and oxidative stress in various models $(26,27)$. In contrast, another study showed that the knockdown of FABP4 upregulates the intracellular ROS levels in adipocytes (14). The discrepancy may be due to different cell models, and therefore, further investigation is needed. In the present study, it was found that the antioxidant effects of miR-455 could be mimicked by downregulation of FABP4 and abrogated by overexpression of FABP4. Therefore, our study implies that targeting of FABP4 may be an effective strategy against oxidative stress in the treatment of endometriosis.

Taken together, our study provides new insight into the mechanisms by which miR-455 alleviates oxidative stress in HESCs. Additionally, we identified FABP4 as a direct target of miR-455. Hence, these results provide strong evidence that miR-455 may be an effective target for the treatment of endometriosis.

\section{Acknowledgements}

Not applicable.

\section{Funding}

This study was supported by a grant from the Natural Foundation of Ningbo Science and Technology Bureau, China (grant no. 2018A610323).

\section{Availability of data and materials}

The datasets used during the present study are available from the corresponding author upon reasonable request.

\section{Authors' contributions}

WT and OC performed experiments; FY performed the statistical analysis of the data; LC designed the study and drafted 
the manuscript. All authors read and approved the manuscript and agree to be accountable for all aspects of the research in ensuring that the accuracy or integrity of any part of the work are appropriately investigated and resolved.

\section{Ethics approval and consent to participate}

Not applicable.

\section{Patient consent for publication}

Not applicable.

\section{Competing interests}

The authors state that they have no competing interests.

\section{References}

1. Kennedy S, Bergqvist A, Chapron C, D'Hooghe T, Dunselman G Greb R, Hummelshoj L, Prentice A and Saridogan E; ESHRE Special Interest Group for Endometriosis and Endometrium Guideline Development Group: ESHRE guideline for the diagnosis and treatment of endometriosis. Hum Reprod 20 2698-2704, 2005.

2. Sampson JA: Metastatic or embolic endometriosis, due to the menstrual dissemination of endometrial tissue into the venous circulation. Am J Pathol 3: 93-110.43, 1927.

3. Sourial S, Tempest N and Hapangama DK: Theories on the pathogenesis of endometriosis. Int J Reprod Med 2014: 179515, 2014.

4. Vinatier D, Orazi G, Cosson M and Dufour P: Theories of endometriosis. Eur J Obstet Gynecol Reprod Biol 96: 21-34, 2001.

5. Christodoulakos G, Augoulea A, Lambrinoudaki I, Sioulas V and Creatsas G: Pathogenesis of endometriosis: The role of defective 'immunosurveillance'. Eur J Contracept Reprod Health Care 12: 194-202, 2007.

6. Bartel DP: MicroRNAs: Genomics, biogenesis, mechanism, and function. Cell 116: 281-297, 2004.

7. Qin SB, Peng DY, Lu JM and Ke ZP: miR-182-5p inhibited oxidative stress and apoptosis triggered by oxidized low-density lipoprotein via targeting toll-like receptor 4. J Cell Physiol 233: 6630-6637, 2018.

8. Diao H, Liu B, Shi Y, Song C, Guo Z, Liu N, Song X, Lu Y, Lin X and Li Z: MicroRNA-210 alleviates oxidative stress-associated cardiomyocyte apoptosis by regulating BNIP3. Biosci Biotechnol Biochem 81: 1712-1720, 2017.

9. Hou M, Zuo X, Li C, Zhang Y and Teng Y: Mir-29b regulates oxidative stress by targeting SIRT1 in ovarian cancer cells. Cell Physiol Biochem 43: 1767-1776, 2017.

10. Zhang $\mathrm{S}$, Wu W, Jiao G, Li C and Liu H: miR-455-3p activates Nrf2/ARE signaling via HDAC2 and protects osteoblasts from oxidative stress. Int J Biol Macromol 107: 2094-2101, 2018.

11. Xu D, Zhu H, Wang C, Zhu X, Liu G, Chen C and Cui Z: microRNA-455 targets cullin 3 to activate Nrf2 signaling and protect human osteoblasts from hydrogen peroxide. Oncotarget 8: 59225-59234, 2017.
12. Furuhashi M and Hotamisligil GS: Fatty acid-binding proteins: Role in metabolic diseases and potential as drug targets. Nat Rev Drug Discov 7: 489-503, 2008.

13. Steen KA, Xu H and Bernlohr DA: FABP4/aP2 regulates macrophage redox signaling and inflammasome activation via control of UCP2. Mol Cell Biol 37: pii: e00282-16, 2017.

14. Kajimoto K, Minami Y and Harashima H: Cytoprotective role of the fatty acid binding protein 4 against oxidative and endoplasmic reticulum stress in 3T3-L1 adipocytes. FEBS Open Bio 4: 602-610, 2014.

15. Livak KJ and Schmittgen TD: Analysis of relative gene expression data using real-time quantitative PCR and the 2(-Delta Delta C(T)) method. Methods 25: 402-408, 2001.

16. Xu Y, Wang W, Jin K, Zhu Q, Lin H, Xie M and Wang D: Perillyl alcohol protects human renal tubular epithelial cells from hypoxia/reoxygenation injury via inhibition of ROS, endoplasmic reticulum stress and activation of PI3K/Akt/eNOS pathway. Biomed Pharmacother 95: 662-669, 2017.

17. Tsukahara H: Biomarkers for oxidative stress: Clinical application in pediatric medicine. Curr Med Chem 14: 339-351, 2007.

18. Halliwell B, Clement MV, Ramalingam J and Long LH: Hydrogen peroxide. Ubiquitous in cell culture and in vivo? IUBMB Life 50: 251-257, 2000.

19. Singh M, Sharma $H$ and Singh N: Hydrogen peroxide induces apoptosis in HeLa cells through mitochondrial pathway. Mitochondrion 7: 367-373, 2007.

20. Clement MV, Ponton A and Pervaiz S: Apoptosis induced by hydrogen peroxide is mediated by decreased superoxide anion concentration and reduction of intracellular milieu. FEBS Lett 440: 13-18, 1998.

21. Degterev A, Boyce M and Yuan J: A decade of caspases. Oncogene 22: 8543-8567, 2003.

22. Thornberry NA: Caspases: A decade of death research. Cell Death Differ 6: 1023-1027, 1999.

23. Zal F, Khademi F, Taheri R and Mostafavi-Pour Z: Antioxidant ameliorating effects against $\mathrm{H}_{2} \mathrm{O}_{2}$-induced cytotoxicity in primary endometrial cells. Toxicol Mech Methods 28: 122-129, 2018.

24. Scutiero G, Iannone P, Bernardi G, Bonaccorsi G, Spadaro S, Volta CA, Greco P and Nappi L: Oxidative stress and endometriosis: A systematic review of the literature. Oxid Med Cell Longev 2017: 7265238, 2017.

25. Yan J, Gong Y, She YM, Wang G, Roberts MS and Burczynski FJ Molecular mechanism of recombinant liver fatty acid binding protein's antioxidant activity. J Lipid Res 50: 2445-2454, 2009.

26. Gong Y, Yu Z, Gao Y, Deng L, Wang M, Chen Y, Li J and Cheng B: FABP4 inhibitors suppress inflammation and oxidative stress in murine and cell models of acute lung injury. Biochem Biophys Res Commun 496: 1115-1121, 2018.

27. Rahman N, Jeon M and Kim YS: Methyl gallate, a potent antioxidant inhibits mouse and human adipocyte differentiation and oxidative stress in adipocytes through impairment of mitotic clonal expansion. Biofactors 42: 716-726, 2016.

This work is licensed under a Creative Commons Attribution-NonCommercial-NoDerivatives 4.0 International (CC BY-NC-ND 4.0) License. 